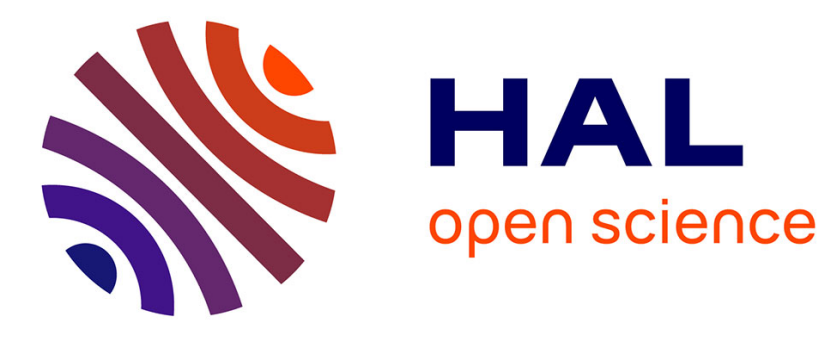

\title{
Synchronization and desynchronization of neural oscillators
}

Arnaud Tonnelier, Sylvain Meignen, Holger Bosch, Jacques Demongeot

\section{To cite this version:}

Arnaud Tonnelier, Sylvain Meignen, Holger Bosch, Jacques Demongeot. Synchronization and desynchronization of neural oscillators. Neural Networks, 1999, 12, pp.1213-1228. hal-00393752

\section{HAL Id: hal-00393752 \\ https://hal.science/hal-00393752}

Submitted on 9 Jun 2009

HAL is a multi-disciplinary open access archive for the deposit and dissemination of scientific research documents, whether they are published or not. The documents may come from teaching and research institutions in France or abroad, or from public or private research centers.
L'archive ouverte pluridisciplinaire HAL, est destinée au dépôt et à la diffusion de documents scientifiques de niveau recherche, publiés ou non, émanant des établissements d'enseignement et de recherche français ou étrangers, des laboratoires publics ou privés. 


\section{Synchronization and Desynchronization of neural oscillators}

Arnaud Tonnelier $\dagger^{*}$ Sylvain Meignen $\dagger$ Holger Bosch $\ddagger$ Jacques Demongeot $\dagger$ †TIMC-IMAG, Faculté de Médecine, Université de Grenoble, France

$\ddagger$ Department of Computer Science, University of Geneva, Switzerland

*E-mail: Arnaud.Tonnelier@imag.fr 


\begin{abstract}
We have used continuous and discrete-time versions of a neural oscillator model to analyze how various types of synaptic connections between oscillators affect synchronization and desynchronization phenomena. First, we present a synthesis of the mathematical properties of both neural oscillator versions. Then, we show that the choice of parameters leads to a relationship between the two versions. Finally, we achieve the coupling of two oscillators in order to study how synaptic connections affect the phase lag. With this in mind, we state some of the results for the continuous-time model. The second part of this paper deals with the behavior of neural networks comprising connected oscillators, wich involves looking at the conditions for desynchronization of a totally synchronized oscillator net. Such a study has been carried out both for a fully and for a sparsely connected network. This leads to the observation that some architectures enable proper desynchronization when the size of the network is large. While searching for the conditions for desynchronization, we have discovered that a macroscopic description of the network is sometimes possible. To conclude, we discuss the advantages and the limitations of this macroscopic approach.
\end{abstract}

Keywords: Dynamical system, Neural oscillators, Synchronization, Desynchronization. 


\section{Introduction}

Oscillatory electrical activities have been uncovered in several brain regions, such as the visual cortex, the olfactory bulb, studied by Li and Hopfield (1989), and the hippocampus (Gluck, 1996). Recordings carried out by Gray et al.(1989) on the first visual cortical area of anesthesized cats have shown that visual stimuli can generate brief, coherent electrical activity. These biological results are consistent with the theoretical investigations of Von der Malsburg (1981), which gave rise to the labelling hypothesis, i.e., cell assemblies are established through oscillations, and labeled by a phase. In accordance with this hypothesis, the electrical activity might be explained by the fact that visual stimuli provoke brief phase locking of separate regions of the cortical area. Our purpose has therefore been to modelize this phenomenon by synchronization of neural oscillators followed by a fast desynchronization.

In order to do this, we were confronted with a choice between the use of continuous or discrete time for the oscillator network. Many people find discrete-time nets to be conceptually simpler, and more appropriate for dealing with discrete or symbolic data. On the other hand, continuous-time nets seem more natural in many models and applications. Since there is no bi-

ological evidence concerning the kind of network to be used, we will try to explore the labelling hypothesis through both continuous and discrete time approaches. To this end, we will use a common type of continuous-time dynamic describing additive nets:

$$
\frac{d x_{i}}{d t}=-c_{i} x_{i}+\sum_{j=1}^{n} W_{i j} g_{j}\left(x_{j}\right),(i=1, \ldots, n)
$$


Here, $g_{j}$ is the activation function of unit $j$; its value is the output of unit $j . c_{i}$ is a relaxation coefficient, $W_{i j}$ is the weight of the connection line from unit $j$ to unit $i$ and $x_{i}(t)$ is the activity of unit $i$. Several investigations have been carried out concerning the synchronization aspect of the labelling hypothesis, using special values of the parameters leading to specific architecture. For a study of a ring network, we may refer to Atiya and Baldi (1989). A more systematic mathematical study is available in Hoppensteadt and Izhikevitch(1997). For discrete-time networks, we will use a general additive net of the form:

$$
y_{i}(t+1)=g_{i}\left(\sum_{j=1}^{n} W_{i j} y_{j}\right),(i=1, \ldots, n)
$$

Here, $y_{i}$ is the output of unit $i$. This model was first introduced by Hopfield (1982). Synchronization for such systems poses a difficult problem, from a mathematical point of view, and, as far as we know, little has been done to pursue this goal. However, synchronization can also be achieved artificially by the addition of inputs, as we will be explained later.

One major aim of our paper is to study desynchronization of additive nets after their synchronization for specific kinds of synaptic weights. To start with, we describe, in section 2 , the basic unit of the network, the neural oscillator. In the following section, we study the mathematical properties of the oscillator, in order to illustrate the differences between continuous and discrete time approaches. In contrast, section 4 focuses on the possible relationship between the two descriptions. In the final section, we analyze the coupling of two oscillators, in order to obtain an insight into the desynchronization of more complex neural nets. 


\section{Models and Equations}

This section is devoted to the presentation of a single oscillator. This comprises two neurons, one excitatory and one inhibitory as depicted in the following interaction diagram:

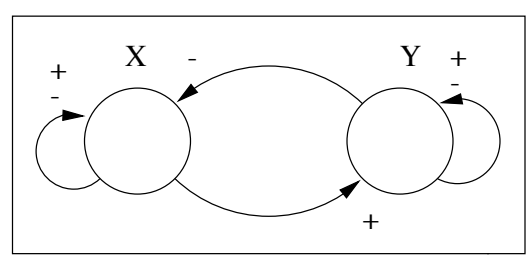

Figure 1: Representation of two coupled oscillators, signs indicate whether the action in the direction of the arrow is inhibitory or excitatory.

$\mathrm{X}$ (or Y) can be either the activity of the excitatory (or inhibitory) neuron, or its output. From now on, $U_{x}(t)$ (or $U_{y}(t)$ ) will denote the output of the excitatory (or inhibitory) neuron and $x(t)$ (or $y(t))$ will represent its activity. Such a modelization was first carried out by Wilson and Cowan (1972), in order to analyze the interaction between a population of inhibitory neurons and a population of excitatory neurons. Furthermore, this kind of interaction diagram has been widely used to interpret many regulated biological systems, particularly in genetic (Thomas and D'Ari 1989). In this paper, we consider two kinds of evolutional behavior. First, we describe a continuous-time evolution for $U_{x}$ and $U_{y}$, given by:

$$
\left\{\begin{array}{l}
\frac{d U_{x}(t)}{d t}=-\frac{U_{x}(t)}{\tau}+\tanh \left(\lambda U_{x}(t)\right)-\tanh \left(\lambda U_{y}(t)\right) \\
\frac{d U_{y}(t)}{d t}=-\frac{U_{y}(t)}{\tau}+\tanh \left(\lambda U_{y}(t)\right)+\tanh \left(\lambda U_{x}(t)\right)
\end{array}\right.
$$


where $\tau$ is a characteristic time constant and $\lambda>0$ is the amplification gain. Such a system is a simple case of additive nets, as described in the introduction, in which synaptic connections are equal to 1 or -1 . Furthermore, the system allows oscillatory behavior for $U_{x}(t)$ and $U_{y}(t)$. Secondly, we also emphasize discrete-time systems owing to the fact that such systems take delays into account naturally (Chapeau-Blondeau and Chauvet, 1992). We therefore study the following Hopfield model for the evolution of $x(t)$ and $y(t)$ :

$$
\left\{\begin{array}{l}
x(t+1)=g\left(a_{1} x(t)+a_{2} y(t)\right) \\
y(t+1)=g\left(a_{3} x(t)+a_{4} y(t)\right)
\end{array}\right.
$$

where $g(y)=\tanh (y)$ and $a_{1}, a_{2}, a_{3}$ and $a_{4}$ are the synaptic weights. The above system is obviously a particular case of the discrete-time evolution system described in the introduction. In accordance with the symmetrical properties of the continuous-time version, but also for the sake of simplicity, we consider that $a_{3}=-a_{2}$ and $a_{4}=a_{1}$. Despite the restrictive nature of this choice, it may prove useful when comparing the two versions of the oscillator. Furthermore, in the present work, the study of neural networks comprising oscillators will show the relevance of this simplification. Thus, $x(t)$ and $y(t)$ are solutions of:

$$
\left\{\begin{array}{l}
x(t+1)=\tanh (\alpha x(t)-\beta y(t)) \\
y(t+1)=\tanh (\beta x(t)+\alpha y(t))
\end{array}\right.
$$




\section{Oscillator properties}

This section is divided into two parts ; the first is devoted to the discrete-time version and the second involves its continuous counterpart. Let us first look at a behavioral analysis of the discrete-time system (2), when $\alpha$ and $\beta$ are varied. Secondly, we will discuss classical results involving the bifurcation study and period calculation for the continuous version, which are also available in Atiya and Baldi (1989). In addition, we will try to shed light on the existence of fixed points. Finally, we will undertake an investigation of isochrons, which make it easier to understand the synchronization process.

\subsection{Discrete-time oscillator}

We have carried out a study of system (2), looking at the following points:

- In property 1 , we seek the conditions that dictate whether the fixed point $(0,0)$ is repulsive or attractive.

- In property 2, we set supplementary conditions for the existence of other fixed points, and we then discuss their stability.

- In property 3 , we calculate an approximation of the period.

Property 1 The origin is a local attractive fixed point if and only if:

$$
\alpha^{2}+\beta^{2}<1
$$


Proof: From dynamical systems theory, it follows that near the origin, the behavior of system (2) is equivalent to:

$$
\left(\begin{array}{l}
x(t+1) \\
y(t+1)
\end{array}\right)=\operatorname{Jac}(0,0)\left(\begin{array}{l}
x(t) \\
y(t)
\end{array}\right)
$$

where $\operatorname{Jac}(0,0)$ is the Jacobian matrix at the origin.

Therefore, the origin is attractive if and only if the spectral radius of the Jacobian matrix at the origin is less than 1 , which reads:

$$
\rho(\operatorname{Jac}(0,0))=\sqrt{\alpha^{2}+\beta^{2}}<1
$$

When $\alpha^{2}+\beta^{2}>1$, the trajectories may tend towards a limit cycle, as shown in Figure 2 (as the rotation angle of trajectories around the cycle is not a rational number, the cycle comprises an infinite number of points). When the dynamic is of this sort, we speak of an oscillator with pacemaker activity, or a pacemaker oscillator.

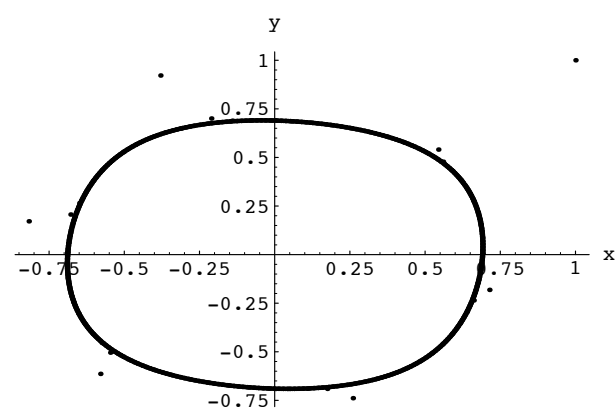

Figure 2: Trajectories in the $(x, y)$ plane for values of $\alpha$ and $\beta$ so that $\alpha^{2}+\beta^{2}>1(\alpha=0.6$ and $\beta=1$ ) in order to obtain a limit cycle. 
From now on, we will refer to secondary Hopf bifurcation as the change in stability of the origin. Beyond the secondary Hopf bifurcation, the dynamic may also have fixed points:

Property 2 The system can have either one, five or nine fixed points.

Proof: A fixed point $(x, y)$ satisfies the equilibrium system (ES):

$$
\left\{\begin{array}{l}
x=\tanh (\alpha x-\beta y) \\
y=\tanh (\beta x+\alpha y)
\end{array}\right.
$$

hence, it also gives:

$$
\left\{\begin{array}{l}
y=\frac{1}{\beta}(\alpha x-\operatorname{argtanh}(x))=f(x) \\
x=\frac{1}{\beta}(-\alpha y+\operatorname{argtanh}(y))=-f(y)
\end{array}\right.
$$

With such a system, three cases may occur: we can have one solution, as depicted in figure 3(A), five solutions, when the two curves are tangential, and, possibly, nine solutions, as shown in figure $3(\mathrm{~B})$ :
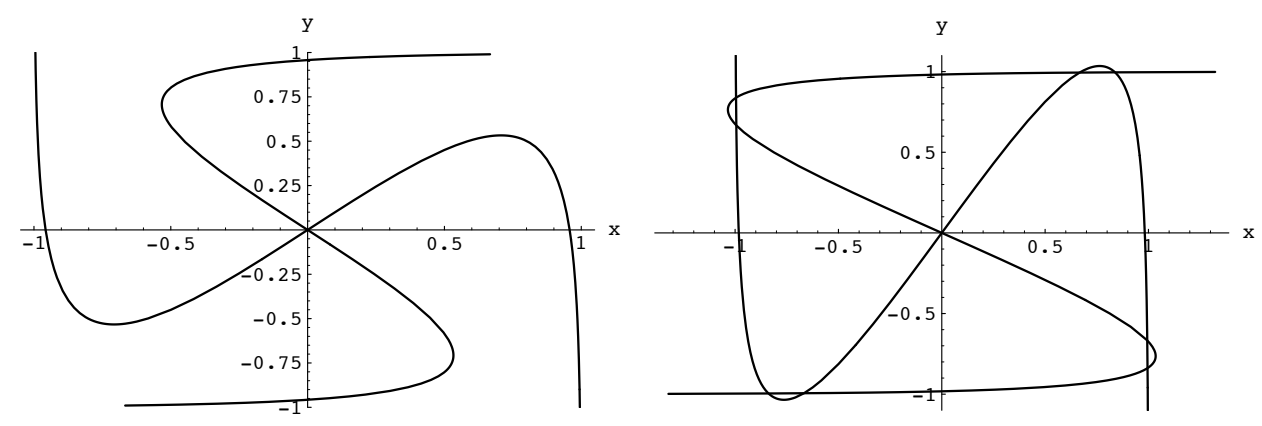

Figure 3: Representation of the nullclines of the discrete model in the $(x, y)$ plane. Their intersections represent the fixed points of the system. In figure (A), the parameters are chosen 
so that there is only one fixed point, whereas, in figure (B), they are chosen so as to obtain nine fixed points.

Furthermore, we have:

$$
f^{\prime}(x)=\frac{1}{\beta}\left(\alpha-\frac{1}{1-x^{2}}\right)
$$

From this we can infer that:

1. if $0 \leq \alpha \leq 1, f$ is a decreasing function, and thus $f \circ f$ is increasing, then $x+f \circ f(x)=0$ has only one solution, $x=0$, which implies that the only fixed point is $(0,0)$

2. if $\alpha>1$

Since $f(x)$ is increasing for $x$ in $\left[0, \sqrt{1-\frac{1}{\alpha}}\right]$, and decreasing for $x$ in $\left[\sqrt{1-\frac{1}{\alpha}}, 1[\right.$ we may deduce that:

- When the nullclines are tangential for some value of $x$ where $x>0$, we have a single fixed point $(x, y)$ where $x>0$ and $y>0$. Furthermore, $x<\sqrt{1-\frac{1}{\lambda}}$. The system has five fixed points because of the symmetry.

- When the nullclines cross for a value of $x>0$, they then cross twice. We thus have two fixed points in the region $x>0$ and $y>0$, leading to nine fixed points because of the symmetry.

Let us assume that there are some fixed points. Of these fixed points, one has coordinates $\left(x_{1}, y_{1}\right)$ that satisfy $y_{1}>x_{1}$. As $x_{1}$ must be positive, we may deduce that $\alpha>\beta$ 
The above demonstration shows that, if $\beta \geq \alpha$ or $\alpha \leq 1$, we have no fixed point apart from the origin. Therefore, if $\alpha$ and $\beta$ also satisfy $\alpha^{2}+\beta^{2}>1$, we are in a cycle configuration. This also explains the dynamic of the oscillator in terms of bifurcation. Indeed, there is a first bifurcation corresponding to the change in stability of the origin. Another bifurcation occurs when a fixed point appears in each quadrant. Numerically, we have observed that it corresponds to a saddle-node on a limit cycle. This point will be developed in a further work.

Let us return to a limit cycle attractor configuration in order to state a simple property concerning the period of the oscillations.

Property 3 Near the secondary Hopf bifurcation the period $T$ is given by the formula:

$$
\begin{gathered}
T=\frac{2 \pi}{\theta} \\
\text { where } \alpha+i \beta=r e^{i \theta}
\end{gathered}
$$

Proof: It should be remembered that the evolution of the discrete-time oscillator at the origin and near the secondary Hopf bifurcation is governed by:

$$
\left\{\begin{array}{l}
x(t+1)=\alpha x(t)-\beta y(t) \\
y(t+1)=\beta x(t)+\alpha y(t)
\end{array}\right.
$$

So, let $z$ be:

$$
z(t)=x(t)+i y(t)
$$

The evolution equation for $z$ is therefore:

$$
z(t+1)=(\alpha+i \beta) z(t)=r e^{i \theta} z(t)
$$


It is obvious that this formula can only be used if $T$ is really close to an integer.

Remark: The choice of fixed connection strengths is not biologically relevant. A possible improvement would be to introduce uncertainty in the parameters, i.e., to replace the classical iteration by an iteration of the following kind (studied by Demongeot et al. (1997)):

$$
\left\{\begin{array}{l}
x(t+1)=\tanh _{\epsilon}(\alpha x(t)-\beta y(t)) \\
y(t+1)=\tanh _{\epsilon}(\beta x(t)+\alpha y(t))
\end{array}\right.
$$

where $\tanh _{\epsilon}(u)$ is defined by: $\exists \eta \in[-\epsilon, \epsilon] / \tanh _{\epsilon}(u)=\frac{1-\epsilon^{2(1+\eta) u}}{1+\epsilon^{2(1+\eta) u}}$

A possible description of the uncertainty involves making a random choice $\eta \in[-\epsilon, \epsilon]$ at each iteration. This allows us, at each step, to compute the classical iteration with $\alpha=\alpha_{\eta}=(1+\eta) \alpha$ and $\beta=\beta_{\eta}=(1+\eta) \beta$. Figure 4 shows this iteration for two values of $\epsilon$.

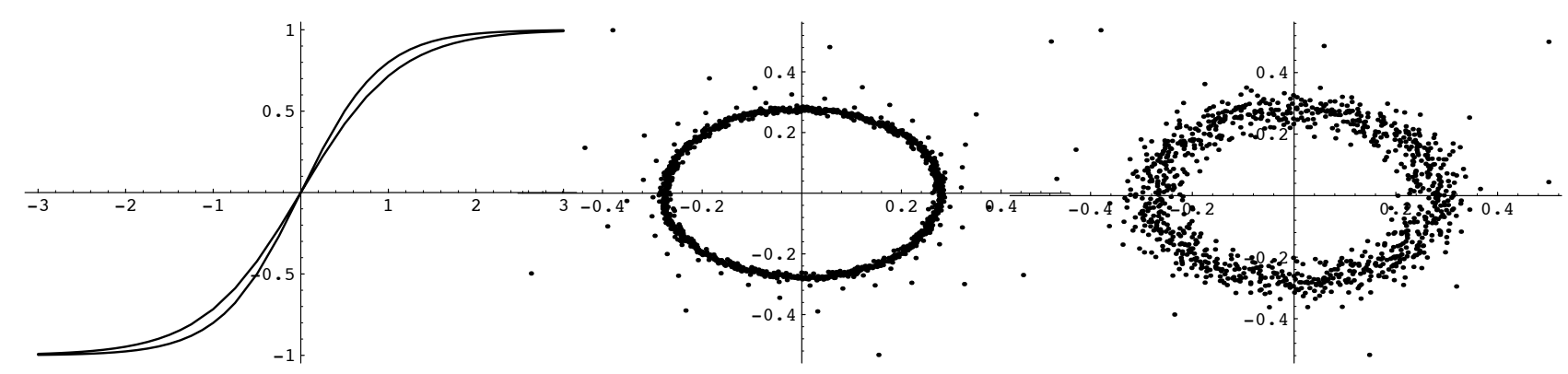

Figure 4: Figure (A) shows a sigmoïdal transfer function delimited by the two maps $\tanh ((1-$ $\epsilon) u$ ) and $\tanh ((1+\epsilon) u)$. Figure $(B)$ shows the trajectories for a compact set valued iteration defined by a random value $\eta$ in $[-\epsilon, \epsilon], \epsilon=0.01$, and :

$$
\left\{\begin{array}{l}
x(t+1)=\tanh ((1+\eta)(\alpha x(t)-\beta y(t))) \\
y(t+1)=\tanh ((1+\eta)(\beta x(t)+\alpha x(t)))
\end{array}\right.
$$


Figure (C) concerns the case for $\epsilon=0.05$.

These phase diagrams show the structural stability of the iterations. The relevance of such a desynchronization model will be discussed at the end of the paper.

\subsection{Continuous-time oscillator}

Here is a reminder of the evolution of $\left(U_{x}(t), U_{y}(t)\right)$ :

$$
\left\{\begin{array}{l}
\frac{d U_{x}(t)}{d t}=-\frac{U_{x}(t)}{\tau}+\tanh \left(\lambda U_{x}(t)\right)-\tanh \left(\lambda U_{y}(t)\right) \\
\frac{d U_{y}(t)}{d t}=-\frac{U_{y}(t)}{\tau}+\tanh \left(\lambda U_{y}(t)\right)+\tanh \left(\lambda U_{x}(t)\right)
\end{array}\right.
$$

As with the discrete-time model, we first focus on the Hopf bifurcation characterization and period calculation. We then discuss the existence of fixed points. The part devoted to isochrons is of major importance in the understanding of the synchronization of oscillators.

\subsubsection{The Hopf bifurcation}

The Hopf bifurcation characterizes the change in dynamic for $\left(U_{x}(t), U_{y}(t)\right)$. The evolution system changes from a single global attractor, the origin, to a cycle attractor. A mathematical criterion has been found out for this transition:

- If $\lambda \tau \leq 1$, the origin is the only stationary point. The origin is stable and all the trajectories converge to the origin (no limit cycles).

- If $\lambda \tau>1$, the origin is unstable and all trajectories converge to a limit cycle attractor. 
Proof for this theorem may be found in Atiya and Baldi (1989).

\subsubsection{Period}

In the case of small oscillations ( $\lambda \tau$ close to 1$)$, the period, $\mathrm{T}$, can be approximated using the imaginary part of the eigenvalues of $\operatorname{Jac}(0,0)$, where $\operatorname{Jac}(0,0)$ is the Jacobian matrix of the system at the origin. This yields $T \approx \frac{2 \pi}{\lambda} \approx 2 \pi \tau$. This approximation is based on a linearization around the origin. As we move further away from the bifurcation point, $\lambda \tau=1$, the first approximation of the period remains fairly good, whereas the second deteriorates more drastically.

\subsubsection{Nullclines}

For all values of $\lambda$ and $\tau$, the origin is the only fixed point. Indeed, as the nullclines are determined by the following equations:

$$
\left\{\begin{array}{l}
U_{y}=-U_{x}+2 \tau \tanh \left(\lambda U_{x}\right) \\
U_{x}=U_{y}-2 \tau \tanh \left(\lambda U_{y}\right)
\end{array}\right.
$$

we can show that $(0,0)$ is the only solution of this system.

Using the following change in variables:

$$
\left\{\begin{array}{l}
U_{x}=\tau(x+y) \\
U_{y}=\tau(x-y)
\end{array}\right.
$$


we seek the solutions of the system:

$$
\left\{\begin{array}{l}
y=-x+\mu \operatorname{argtanh}(x) \\
x=y-\mu \operatorname{argtanh}(y)
\end{array}\right.
$$

where $\mu=\frac{1}{\lambda \tau},-1<x<1$ and $-1<y<1$.

- if $\mu \geq 1$

If $x>0$, then $y>0$, from the first equation. But if $y>0$, then $x<0$, from the second equation which leads to a contradiction. A similar result can be derived for $x<0$, and therefore $x=y=0$ is the only fixed point.

\section{- $\underline{\text { if } \mu<1}$}

We show that every half curve is located in a different region of the plane defined by the tangents at the origin $(y=(\mu-1) x$ and $x=(1-\mu) y)$, i.e., the half curve defined by:

$$
\left\{\begin{array}{c}
y=-x+\mu \operatorname{argtanh}(x) \\
x \in] 0,1[ \\
y \in]-1,1[
\end{array}\right.
$$

satisfies $y-(\mu-1) x>0$ and $x-(1-\mu) y>0$. Similar results may be obtained by rotating the half curve under consideration through $k \frac{\pi}{2}$.

Firstly, $y-(\mu-1) x>0$ since $x \rightarrow-x+\mu \operatorname{argtanh}(x), x \in[0,1[$ is convex. Secondly, the study of $g(x)=x-(1-\mu) y=(2-\mu) x-\mu(1-\mu) \operatorname{argtanh}(x)$ leads to $g(x) \geq 0, \forall x \in\left[0, x_{1}\right]$. We can then show that $y\left(x_{1}\right)=\frac{x_{1}}{1-\mu}>1$, since $g(1-\mu)>0$. Furthermore, studying the variations of $y(x)$, it is found to be increasing on $\left[x_{1}, 1\right.$, which gives $y(x)>1$ if $x \geq x_{1}$. 
The half curve under consideration does not exist on $\left[x_{1}, 1[\right.$, and therefore $g(x)>0$. This ends the demonstration.

\subsubsection{Isochrons}

The use of isochrons to interpret biological phenomena was introduced by Winfree (1974). The first mathematical study of them dates back to Guckenheimer (1975). Isochron properties have been used to study nervous systems by both Pham Dinh et al. (1983), and by Ermentrout and Kopell (1994). For our study, we only need a general definition, which may be stated thus: if two initial conditions are part of the same isochron, then the solutions have identical asymptotic behavior.

Some isochrons of the continuous-time oscillator are shown in Figure 5.

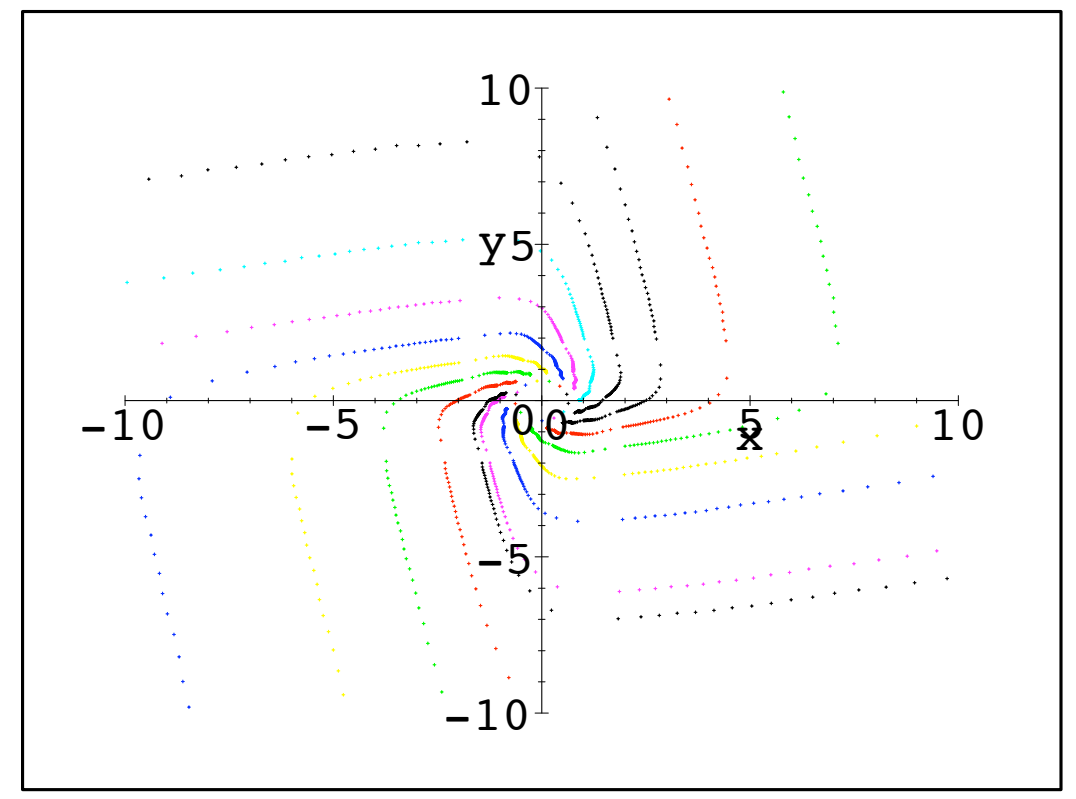

Figure 5: Representation of some isochrons in the $\left(U_{x}, U_{y}\right)$ plane when $\lambda \tau>1$. 
Analysis of isochrons Isochrons of the neural oscillator follow a regular pattern resembling rectangular spirals. The main reasons for this shape are the symmetry of the system and its linearity for large values of $U_{x}$ and $U_{y}$ :

Property 4 For $\left|U_{x}\right|,\left|U_{y}\right|>\frac{2}{\lambda}$, the system can be approximated using the following linear systems:

$$
\begin{array}{ccc}
U_{x}>\frac{2}{\lambda}, \quad U_{y}>\frac{2}{\lambda}: \frac{d U_{x}}{d t} \approx-\frac{U_{x}}{\tau}, & \frac{d U_{y}}{d t} \approx-\frac{U_{y}}{\tau}+2 \\
U_{x}>\frac{2}{\lambda}, U_{y}<-\frac{2}{\lambda}: \frac{d U_{x}}{d t} \approx-\frac{U_{x}}{\tau}+2, & \frac{d U_{y}}{d t} \approx-\frac{U_{y}}{\tau} \\
U_{x}<-\frac{2}{\lambda}, \quad U_{y}>\frac{2}{\lambda}: \frac{d U_{x}}{d t} \approx-\frac{U_{x}}{\tau}-2, & \frac{d U_{y}}{d t} \approx-\frac{U_{y}}{\tau} \\
U_{x}<-\frac{2}{\lambda}, & U_{y}<-\frac{2}{\lambda}: \quad \frac{d U_{x}}{d t} \approx-\frac{U_{x}}{\tau}, & \frac{d U_{y}}{d t} \approx-\frac{U_{y}}{\tau}-2
\end{array}
$$

Proof: For $\left|U_{x}\right|,\left|U_{y}\right|>\frac{2}{\lambda} ;\left|\tanh \left(\lambda U_{x}\right)\right| \approx\left|\tanh \left(\lambda U_{y}\right)\right| \approx 1$. Hence, we obtain the above approximations.

Since the four systems are identical through rotations of $\frac{\pi}{2}$, it is sufficient to study the isochrons in the first quadrant. Figure 6 shows two graphs that will make it easier to understand the following demonstration:
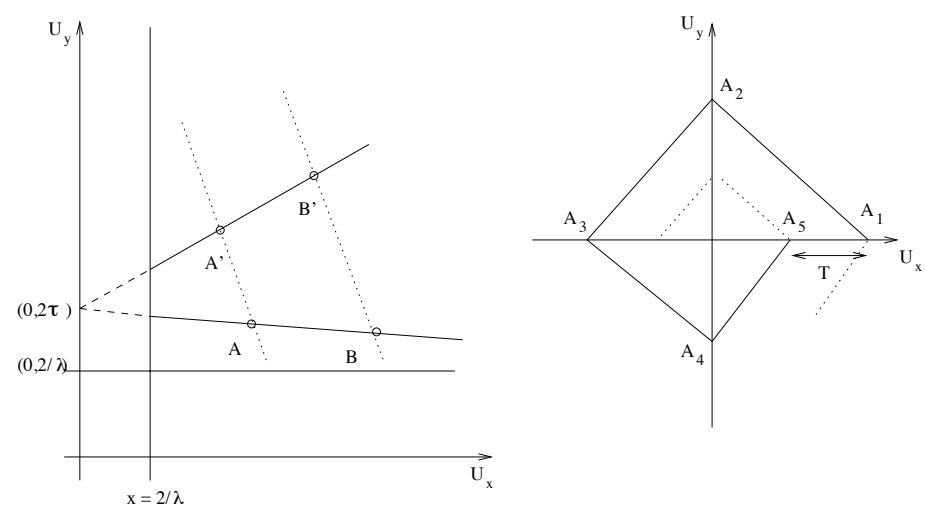

Figure 6: Figure(A) shows two portions, $A A^{\prime}$ ' and BB', of isochrons far beyond the point $\left(\frac{2}{\lambda}, \frac{2}{\lambda}\right)$; thus the isochrons are drawn as lines. $A$ and $A$ ' represent two points located on the 
same isochron, as do B and B'. Details of the geometrical shape are given in the demonstration. Figure $(B)$ shows the drawing of an isochron far beyond $\left(\frac{2}{\lambda}, \frac{2}{\lambda}\right)$ during a complete period.

\section{Demonstration of the shape of the isochrons}

For $U_{x}>\frac{2}{\lambda}$ and $U_{y}>\frac{2}{\lambda}$

Trajectories in the phase space follow: $U_{y}=k U_{x}+2 \tau(k \in \mathbb{R})$. Let us assume that part of an isochron is segment AA'. Let us then consider another isochron crossing the trajectories from A and from A' at B and B', respectively. We would like to show that (AA') is parallel to (BB'). Let us denote a1,a1',b1 and b1' as the respective abscissae of A, A',B and B'. Using the evolution equation of $U_{x}$, and given that the system requires the same time to go from A to A' as to go from B to B', it follows that:

$\ln (\mathrm{b} 1)-\ln (\mathrm{a} 1)=\ln \left(\mathrm{b}^{\prime}\right)-\ln \left(\mathrm{a} 1^{\prime}\right)$ which is equivalent to $\frac{b 1}{a 1}=\frac{b 1^{\prime}}{a 1^{\prime}} \Rightarrow\left(\mathrm{BB}^{\prime}\right) / /\left(\mathrm{AA}^{\prime}\right)$

Thus, all isochrons are parallel in the four quadrants. We may now investigate how the different parts of the same isochron join on the axes. As the function $F\left(U_{x}, U_{y}\right)=\left(-\frac{U_{x}}{\tau}+\tanh \lambda U_{x}-\right.$ $\left.\tanh \lambda U_{y},-\frac{U_{y}}{\tau}+\tanh \lambda U_{x}+\tanh \lambda U_{y}\right)$ is continuous, the isochrons are also continuous, and therefore the straight segments in the different quadrants of the same isochron join on the axes. Since two points on the same trajectory separated by one time period are part of the same isochron, the resulting pattern forms a rectangular spiral.

For $\left|U_{x}\right|<\frac{2}{\lambda}$ and $\left|U_{y}\right|<\frac{2}{\lambda}$

Here, the mathematical study is much more complicated, but it has been proved by Guckenheimer (1975) that isochrons tend towards the cycle perpendicularly. 
Slope of the spiral Since the linear parts of the isochrons are parallel in each quadrant, the slope of the isochrons is well defined. If the oscillator isochrons can be assumed to be a perfect rectangular spiral, as in Figure 6(B), we obtain:

Property 5 The slope of the isochrons can be approximated by

$$
m=e^{\frac{T}{4 \tau}}
$$

where $T$ is the period of the oscillator.

Proof: On the one hand, the distance between $A_{1}$ and $A_{5}$, in Figure 6(B), is given by the length of the trajectory covered during one period. As the system can be assumed to be linear, if $\left\|A_{i}\right\|$ denotes the distance from the origin to the point $A_{i}$, we obtain $T=\tau\left(\ln \left(\left\|A_{5}\right\|\right)-\ln \left(\left\|A_{1}\right\|\right)\right)$, hence:

$$
e^{\frac{T}{\tau}}=\frac{\left\|A_{5}\right\|}{\left\|A_{1}\right\|}
$$

On the other hand, the above fraction can be expressed as

$$
\frac{\left\|A_{5}\right\|}{\left\|A_{1}\right\|}=m^{4}
$$

Where $m$ is the slope of the isochrons when $U_{x}>0$ and $U_{y}>0$. The result of the property is a direct consequence of the above equalities. 


\section{Relationship between the discrete-time and the continuous-}

\section{time versions}

In this part, we discuss the relationships between the parameters of the two versions. We therefore try to switch from the continuous model to the discrete one by making appropriate approximations. Using an Euler method with a time step equal to $\delta$, the continuous model integrates as follows:

$$
\left\{\begin{array}{l}
U_{x}(t+\delta)=\left(1-\frac{\delta}{\tau}\right) U_{x}(t)+\delta\left(\tanh \left(\lambda U_{x}(t)\right)-\tanh \left(\lambda U_{y}(t)\right)\right) \\
U_{y}(t+\delta)=\left(1-\frac{\delta}{\tau}\right) U_{y}(t)+\delta\left(\tanh \left(\lambda U_{y}(t)\right)+\tanh \left(\lambda U_{x}(t)\right)\right)
\end{array}\right.
$$

We study this system close to the Hopf bifurcation point. It is well known that the size of the cycle shrinks as the values of the parameters approach the bifurcation point. Therefore, with an appropriate value of $\tau$, it is possible to get a cycle, for which the diameter is small compared with $\frac{2}{\lambda}$. Thus, we can make the assumption that the respective values of $U_{x}$ and $U_{y}$ are asymptotically small compared to $\frac{2}{\lambda}$. This leads to the following simplification:

$$
\left\{\begin{array}{l}
U_{x}(t+\delta)=\left(1+\delta \lambda\left(1-\frac{1}{\lambda \tau}\right)\right) U_{x}(t)-\delta \lambda U_{y}(t) \\
U_{y}(t+\delta)=\left(1+\delta \lambda\left(1-\frac{1}{\lambda \tau}\right)\right) U_{y}(t)+\delta \lambda U_{x}(t)
\end{array}\right.
$$

Now, let $n \delta=1$ and $\gamma=1+\delta \lambda\left(1-\frac{1}{\lambda \tau}\right)+i \delta \lambda=1+\frac{\lambda}{n}\left(1-\frac{1}{\lambda \tau}\right)+i \frac{\lambda}{n}$. Then:

$$
\left\{\begin{array}{l}
U_{x}(t+1)=\operatorname{Re}\left(\gamma^{n}\right) U_{x}(t)-\operatorname{Im}\left(\gamma^{n}\right) U_{y}(t) \\
U_{y}(t+1)=\operatorname{Im}\left(\gamma^{n}\right) U_{x}(t)+\operatorname{Re}\left(\gamma^{n}\right) U_{y}(t)
\end{array}\right.
$$

where Re and Im stand for the real part and the imaginary part.

Let us now return to the discrete-time system discussed in section 2, in which we use tanh as 
the synaptic transfer function. If we also hypothesize that $x$ and $y$ are sufficiently small, as $U_{x}(t)=x(t)-y(t)$ and $U_{y}(t)=x(t)+y(t)$, it follows:

$$
\left\{\begin{array}{l}
U_{x}(t+1)=\alpha U_{x}(t)-\beta U_{y}(t) \\
U_{y}(t+1)=\beta U_{x}(t)+\alpha U_{y}(t)
\end{array}\right.
$$

We may therefore deduce a relationship between paramaters for the discrete-time and continuoustime versions of the oscillator, which allows them to behave similarly:

$$
\begin{aligned}
& \alpha=\lim _{n \rightarrow+\infty} \operatorname{Re}\left(\gamma^{n}\right) \\
& \beta=\lim _{n \rightarrow+\infty} \operatorname{Im}\left(\gamma^{n}\right)
\end{aligned}
$$

A simple calculation gives us $\alpha=e^{\lambda-\frac{1}{\tau}} \cos (\lambda)$ and $\beta=e^{\lambda-\frac{1}{\tau}} \sin (\lambda)$.

We conclude from this that the equivalence of the two bifurcation equations corresponds to $\lambda \tau=1$ and $\alpha^{2}+\beta^{2}=1$.

Finally, we can investigate the period of the oscillations. It should be remembered that the period of the continuous-time oscillator equals $\frac{2 \pi}{\lambda}$ whereas it is $\frac{2 \pi}{\theta}$ for the discrete one $(\theta=$ $\arg (\alpha+i \beta))$. Since $\theta=\lambda$, it can be observed that the periods are identical at the bifurcation point.

\section{Coupling two oscillators}

In this section, we will first discuss the problem of an eventual phase lag for oscillators linked by one connection, the dynamic of the oscillators being either continuous or discrete in time. Then, we will discuss the case of two oscillators linked by more than one connection arriving at 
the conclusion that the phase lag is difficult to assess with a discrete-time evolution whereas, it can be derived from a continuous one.

\subsection{One connection}

\subsubsection{Discrete model}

We are going to discuss one excitatory connection between the two excitatory neurons, as shown in the following diagram:

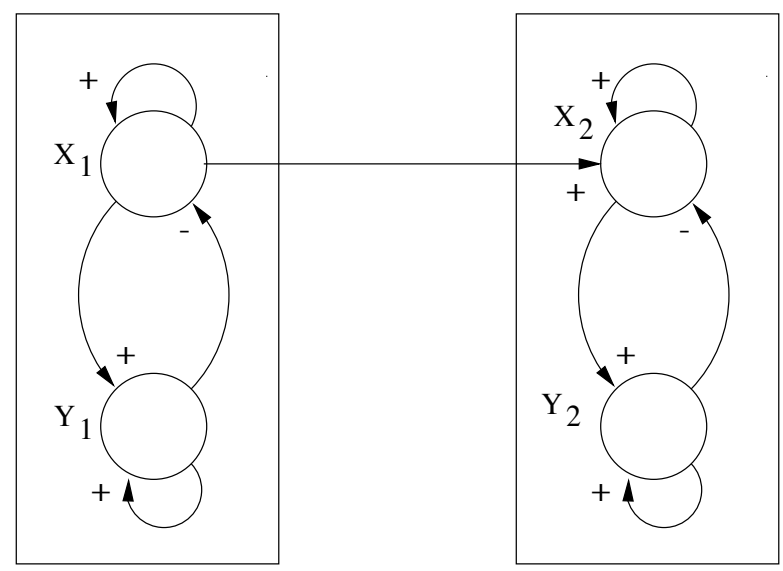

Figure 7: Representation of two modules with a connection between their excitatory neurons. The signs indicate whether the action in the direction of the arrow is inhibitory or excitatory.

Thus, the evolution of $\left(x_{1}, y_{1}\right)$ is unchanged, whereas that of $\left(x_{2}, y_{2}\right)$ is given by:

$$
\left\{\begin{array}{l}
x_{2}(t+1)=\tanh \left(\alpha x_{2}(t)-\beta y_{2}(t)+\mathrm{c} x_{1}(t)\right) \\
y_{2}(t+1)=\tanh \left(\beta x_{2}(t)+\alpha y_{2}(t)\right)
\end{array}\right.
$$

where $c>0$.

At this stage, we have only carried out numerical simulations in which we have chosen $\beta>\alpha$, 
which means that the effect of one neuron on the other is greater than that exerted by the latter neuron on itself. Furthermore, when such a choice is made, the dynamic of an isolated oscillator presents no fixed points (see section 3.1). We expect that the dynamic described by $\left(x_{2}, y_{2}\right)$ behaves similarly when $\mathrm{c}$ is small (this can be checked numerically). Let us denote $p(t)$ the phase lag between the oscillators at time $t$. We investigate the evolution of $(p(t), p(t+1))$ versus the synaptic weight, $c$. For a wide range of values of $c$, the phase lag between each oscillator is quasi-periodic (it might be periodic for special values of c); a limit cycle appears. If we proceed further, and study the evolution of this phase lag as a function of connection $c$, it appears that the stronger the connection, the bigger the cycle. This fact may be explained by a change in the frequency of one of the two connected oscillators: this change is all the greater since $\mathrm{c}$ is large. Figure 8 displays phase diagrams $(p(t), p(t+1))$ for several values of $\mathrm{c}$ (it is important to note that we are careful to maintain a pacemaker activity for both oscillators, 
whatever the value of parameter $\mathrm{c}$ ).
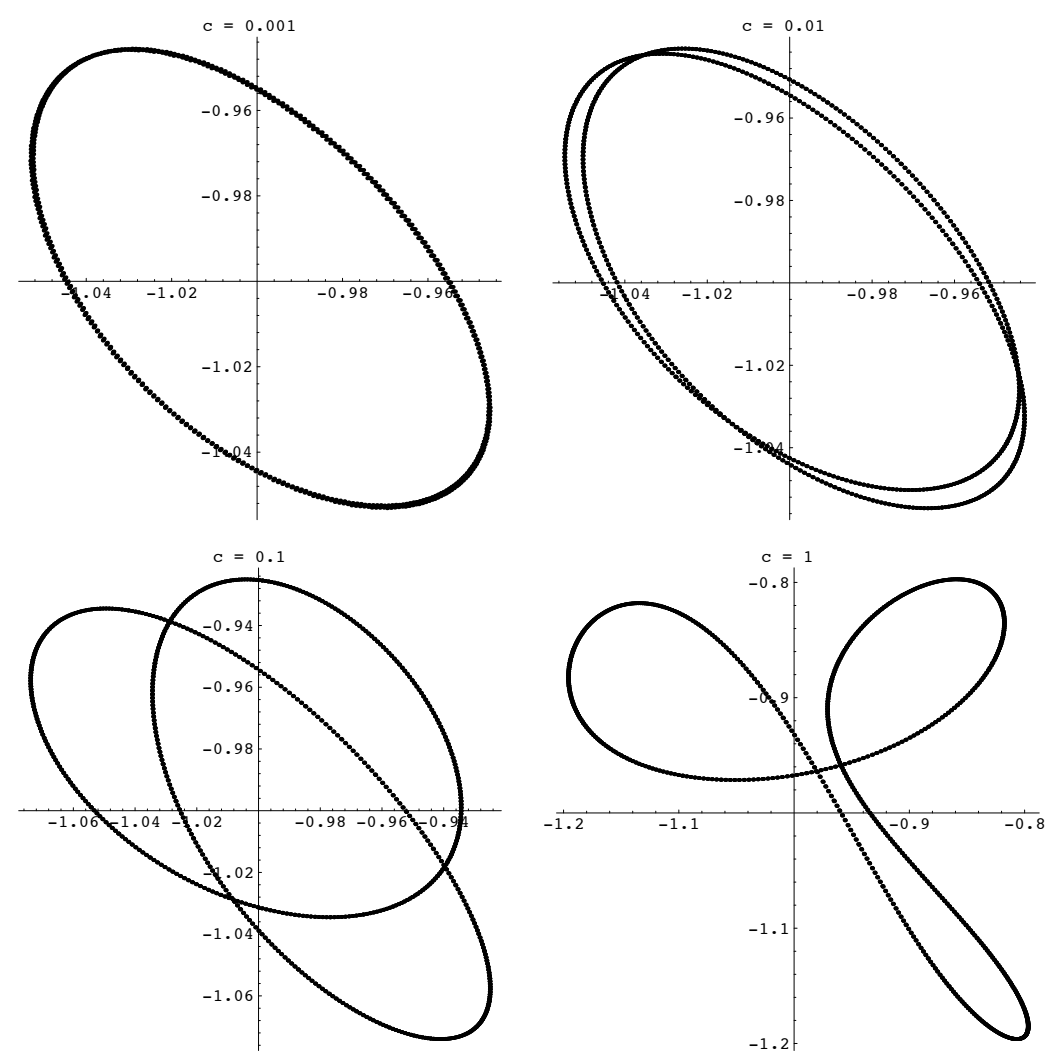

Figure 8: Figure $(A)$ shows the phase diagram $(p(t), p(t+1))$ for a connection strength equal to $0.001, \alpha=0.6$ and $\beta=1$. Figure $(B)$ is similar to figure (A) for a connection strength equal to $0.01, \alpha=0.6$ and $\beta=1$. In figure $(C)$, the connection strength is equal to $0.1, \beta$ with $\alpha$ remaining unchanged. In figure (D), the connection strength is equal to $1, \beta$ and $\alpha$ remaining unchanged.

Throughout the simulations, it appears that such a behavior may easily be reproduced for any other kind of connection. 


\subsubsection{Continuous-time version}

Here, as with the discrete-time system, the excitatory neurons are coupled by a uni-directional excitatory connection. The evolution of the first oscillator remains unchanged, and that of the second gives:

$$
\left\{\begin{array}{l}
\frac{d U_{x_{2}}(t)}{d t}=-\frac{U_{x_{2}}(t)}{\tau}+\tanh \left(\lambda U_{x_{2}}(t)\right)-\tanh \left(\lambda U_{y_{2}}(t)\right)+\tanh \left(c U_{x_{1}}(t)\right) \\
\frac{d U_{y_{2}}(t)}{d t}=-\frac{U_{y_{2}}(t)}{\tau}+\tanh \left(\lambda U_{y_{2}}(t)\right)+\tanh \left(\lambda U_{x_{2}}(t)\right)
\end{array}\right.
$$

where $c>0$.

Simulations show that the phase lag between the oscillators converges to a constant, $d$. Furthermore, $d$ depends neither on the initial conditions of the system, nor on $\lambda$ and $\tau$. The coupling strength, $c$, only affects the time needed to establish the asymptotic phase lag. The value of $d$ approaches $T / 8$ if the system parameters approach the bifurcation point $\lambda \tau=1$, where $T$ is the period of the system. The analysis of system (3) makes it possible to determine analytically the effect of any possible connection between two units of two different oscillators on their phase lag. Details are given in the following property:

Property 6 If we assume that the phase lag induced by the first oscillator on the second converges to a constant value, $d$, the phase lag induced by any other type of connection equals one of the values, $\pm d+k T / 8$ where $k \in\{1,3,5,7\}$, provided that the strength of this connection is low. 
Proof: Let us first consider an oscillator $\left(U_{x}, U_{y}\right)$, evolving as follows

$$
\left\{\begin{array}{l}
\frac{d U_{x}(t)}{d t}=-\frac{U_{x}(t)}{\tau}+\tanh \left(\lambda U_{x}(t)\right)-\tanh \left(\lambda U_{y}(t)\right) \\
\frac{d U_{y}(t)}{d t}=-\frac{U_{y}(t)}{\tau}+\tanh \left(\lambda U_{y}(t)\right)+\tanh \left(\lambda U_{x}(t)\right)
\end{array}\right.
$$

The invariance shown when the vector field is rotated through $\frac{\pi}{2}$ gives:

$$
\begin{aligned}
& \text { 1) } U_{x}(t)=U_{y}\left(t+\frac{T}{4}\right) \\
& \text { 2) }-U_{x}(t)=U_{x}\left(t+\frac{T}{2}\right) \\
& \text { 3) }-U_{y}(t)=U_{y}\left(t+\frac{T}{2}\right)
\end{aligned}
$$

If we consider that the excitatory connection, $c$, in $(3)$, is small, those relationships are still valid for the excited oscillator. It is, therefore, possible to imply the following:

- If a connection is replaced by an identical connection, but in the opposite direction, the phase difference is replaced by its opposite value, i.e., $d *=-d \bmod T$

- if a connection changes its sign, i.e., an excitatory connection becomes inhibitory or vice versa, the phase difference is increased by $\frac{T}{2}$, i.e., $d *=d+\frac{T}{2} \bmod T$

- If a connection originated from the excitatory neuron is replaced by an identical connection originating from the inhibitory neuron, the phase lag is increased by $\frac{T}{4}$, i.e., $d *=d+\frac{T}{4}$ $\bmod T$

- If a connection targeting the excitatory neuron is replaced by an identical connection targeting the inhibitory neuron, the phase difference is decreased by $\frac{T}{4}$, i.e., $d *=d-\frac{T}{4}$ $\bmod T$ 
The phase difference induced by any connection is obtained by applying one or more of the above rules.

\subsection{Several connections}

\subsubsection{Discrete model}

The connections between the oscillators are as follows:

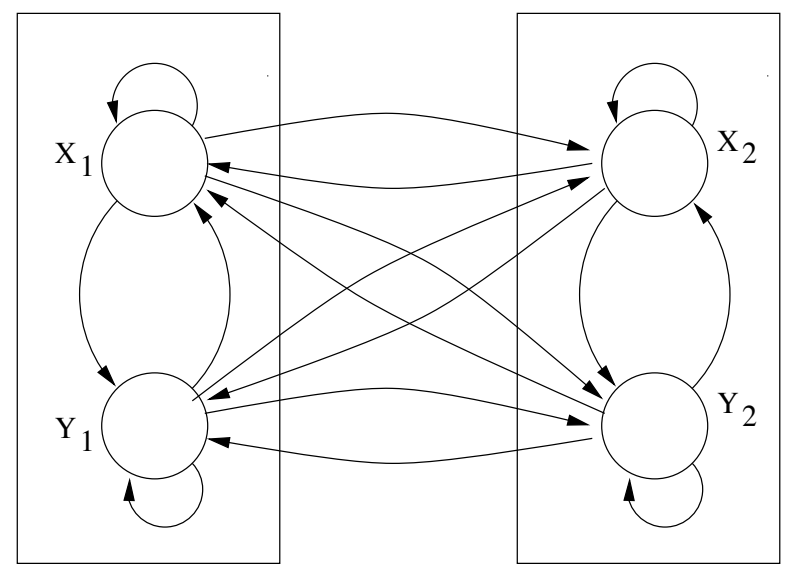

Figure 9: Representation of two completely connectd modules; an arrow represents a connection between two neurons. The action of one neuron to another is not specified.

Simulations are made for the connection strength evolving in $\left[c_{\min }, c_{\max }\right]$. When $c_{\min }$ equals $c_{\max }$, the oscillators tend to synchronize. In contrast, if $c_{\min }$ is different from $c_{\max }$, the phase lag between oscillators, $p(t)$, is such that map $p(t+1)$ versus $p(t)$ is a cycle. Numerically, we may observe that the bigger the difference between $c_{\max }$ and $c_{\min }$, the bigger this cycle. The dynamic properties of the system are similar to these obtained with one connection; $(p(t), p(t+1))$ tends towards the cycle exponentially faster when the connection strength increases. 


\subsubsection{Continuous model}

The evolution system has the following form:

$$
\left\{\begin{array}{l}
\frac{d U_{x_{i}}(t)}{d t}=-\frac{U_{x_{i}}(t)}{\tau}+\tanh \left(\lambda U_{x_{i}}(t)\right)-\tanh \left(\lambda U_{x_{i}}(t)\right)+\sum_{i \neq j} \tanh \left(c_{x_{i} x_{j}} U_{x_{j}}(t)+c_{x_{i} y_{j}} U_{y_{j}}(t)\right) \\
\frac{d U_{y_{i}}(t)}{d t}=-\frac{U_{y_{i}}(t)}{\tau}+\tanh \left(\lambda U_{y_{i}}(t)\right)+\tanh \left(\lambda U_{x_{i}}(t)\right)+\sum_{i \neq j} \tanh \left(c_{y_{i} x_{j}} U_{x_{j}}(t)+c_{y_{i} y_{j}} U_{y_{j}}(t)\right)
\end{array}\right.
$$

where $i$ and $j$ are equal to 1 or 2 . If the coupling between two oscillators comprises several connections, simulations show that the phase lag is still asymptotically stable. If the oscillators are near the bifurcation point, the connections can be classified into four classes, according to the phase lag they induce. Connections inducing opposite phase lags ( $T / 8$ is opposite to $5 T / 8$, and $3 T / 8$ is opposite to $7 T / 8$ ) anneal each other and the total effect of these connections is negligible. The weighted mean value of the remaining connections equals the phase lag induced by the set of connections. In particular, if all connections anneal each other, the oscillators act almost independently, and the phase lag depends on the initial conditions of the system.

Hoppensteadt and Izhikevich (1997) have also studied this kind of problem using their "canonical" model, which has given similar results. They have found that with the appropriate connections, the phase lag can be monitored, and, in addition, they have shown that some connection diagrams enable the oscillators to behave independently.

\section{Network of connected oscillators}

We have just shown that the phase lag between two oscillators may be monitored using a continuous-time evolution, but no such conclusion is valid for the discrete-time model, even 
close to the bifurcation point. Nevertheless, as the number of oscillators increases, the phase lag between oscillators can no longer be assessed using continuous-time evolution. For this reason, synchronization is achieved by means of external stimuli. Using discrete-time evolution systems, we have investigated the desynchronization aspect of the labelling hypothesis. Such a study could also have been carried out with the continuous-time version.

\subsection{Dynamic of the network}

We now consider a network comprising $\mathrm{N}$ oscillators, whose dynamic gives:

$$
\left\{\begin{array}{l}
x_{i}(t+1)=a_{i} \tanh \left\{\alpha_{i} x_{i}(t)-\beta_{i} y_{i}(t)+\sum_{j=1, j \neq i}^{N}\left(c_{x_{i} x_{j}} x_{j}(t)+c_{x_{i} y_{j}} y_{j}(t)\right)\right\} \\
y_{i}(t+1)=a_{i} \tanh \left\{\beta_{i} x_{i}(t)+\alpha_{i} y_{i}(t)+\sum_{j=1, j \neq i}^{N}\left(c_{y_{i} x_{j}} x_{j}(t)+c_{y_{i} y_{j}} y_{j}(t)\right)\right\}
\end{array}\right.
$$

where $i \in\{1 . . N\}$ and $\left(x_{i}, y_{i}\right)$ describes the electrical response of the excitatory and inhibitory neurons for the $i^{\text {th }}$ oscillator. The synaptic weights follow Dale's principle, i.e., $c_{y x}$ and $c_{x x}$ are both positive, and, $c_{x y}$ and $c_{y y}$ are both negative. Moreover, a simple change in variable makes it possible to consider $a_{i}$ as being equal to 1 . At a given time, t, we apply an input characterized by $(r, \theta)$, to each oscillator, giving the following dynamic:

$$
\left\{\begin{array}{l}
x_{i}(t+1)=\tanh \left\{\alpha_{i} x_{i}(t)-\beta_{i} y_{i}(t)+\sum_{j=1, j \neq i}^{N}\left(c_{x_{i} x_{j}} x_{j}(t)+c_{x_{i} y_{j}} y_{j}(t)\right)\right\}+r \cos (\theta) \\
y_{i}(t+1)=\tanh \left\{\beta_{i} x_{i}(t)+\alpha_{i} y_{i}(t)+\sum_{j=1, j \neq i}^{N}\left(c_{y_{i} x_{j}} x_{j}(t)+c_{y_{i} y_{j}} y_{j}(t)\right)\right\}+r \sin (\theta)
\end{array}\right.
$$


the next stages are calculated as follows:

$$
\left\{\begin{array}{l}
x_{i}(t+1)=\tanh \left\{\alpha_{i} x_{i}(t)-\beta_{i} y_{i}(t)+\sum_{j=1, j \neq i}^{N}\left(c_{x_{i} x_{j}} x_{j}(t)+c_{x_{i} y_{j}} y_{j}(t)\right)\right\} \\
y_{i}(t+1)=\tanh \left\{\beta_{i} x_{i}(t)+\alpha_{i} y_{i}(t)+\sum_{j=1, j \neq i}^{N}\left(c_{y_{i} x_{j}} x_{j}(t)+c_{y_{i} y_{j}} y_{j}(t)\right)\right\}
\end{array}\right.
$$

The aim of such an input is to synchronize the network, in order to study its resulting behavior, especially its desynchronization. Furthermore, we require two other major properties. Firstly, in the absence of stimulation, the activity of the network must remain low, which means that the network is naturally well desynchronized. Secondly, the network must have at least one pacemaker oscillator, which means that the vector $(0, \ldots, 0)$ is not an attractor for the network characterized by $\left(x_{1}, y_{1}, \ldots, x_{N}, y_{N}\right)$. We will see that when the above conditions are fulfilled, the resulting behavior is that of a desynchronized network comprising pacemaker oscillators, regardless of the values of $r$ and $\theta$.

\subsection{Different kinds of architectures}

Many articles discuss neural networks with simple architectures such as the "ring network" (Pasemann, 1995) or the chain of oscillators (Wang, 1996), giving rise to simple mathematical studies. In contrast, we have chosen to impose the weakest possible conditions on connections. This approach does not allow us to carry out exact calculations and we must therefore often make mathematically relevant approximations. 


\subsubsection{Definitions}

For the purposes of our study, we introduce two other entities in order to characterize the state of the network. We refer to the following entities as the mean activity of the excitatory and the mean activity of the inhibitory neurons, respectively:

$$
\begin{aligned}
& \mathbf{E}(t)=\frac{1}{N} \sum_{i=1}^{N} x_{i}(t) \\
& \mathbf{I}(t)=\frac{1}{N} \sum_{i=1}^{N} y_{i}(t)
\end{aligned}
$$

A good test for network desynchronization is $\lim _{t \rightarrow \infty}(\mathbf{E}(t), \mathbf{I}(t))=(0,0)$.

\subsubsection{Fully connected neural network}

Using a fully connected network, we have investigated the conditions needed to fulfill the requirements laid out in section 6.1 . For that purpose, we may make the following assumptions $(\mathrm{H})$ :

1. $c_{x x}=c_{y x}=c_{e}$ and $c_{y y}=c_{x y}=-c_{i}$ where $c_{e}$ and $c_{i}$ are both positive.

2. all oscillators are identical.

3. the network remains near the secondary Hopf bifurcation.

Assuming that $(\mathrm{H})$ holds true, the requirement of a well desynchronized network implies the following: 
Property 7 Let $f_{e}=c_{e}(N-1)$ and $f_{i}=c_{i}(N-1)$ be the excitatory and the inhibitory strengths of the network, respectively.

$$
\begin{aligned}
& \text { Using } \Delta(q)=\left(f_{e}-f_{i}\right)^{2}-4 \beta\left(\beta+f_{e}+f_{i}\right) \text {, we need: } \\
& \qquad\left\{\begin{array}{l}
\text { if } \Delta(q)>0: \max \left(\left|\alpha+\frac{f_{e}-f_{i}}{2}+\frac{1}{2} \sqrt{\Delta(q)}\right|,\left|\alpha+\frac{f_{e}-f_{i}}{2}-\frac{1}{2} \sqrt{\Delta(q)}\right|\right)>1 \\
\text { if } \Delta(q)<0: \alpha^{2}+\beta^{2}+f_{e}(\alpha+\beta)+f_{i}(\beta-\alpha)<1
\end{array}\right.
\end{aligned}
$$

The proof of this property is given in Appendix A.

The requirement for the existence of at least one pacemaker oscillator reads as follows:

Property 8 If the network satisfies property 7 , using $\Delta(p)=4 \beta\left(c_{i}+c_{e}-\beta\right)+\left(c_{e}-c_{i}\right)^{2}$, we need:

$$
\left\{\begin{array}{l}
\text { if } \Delta(p)>0: \max \left(\left|\alpha-\frac{c_{e}-c_{i}}{2}+\frac{1}{2} \sqrt{\Delta(p)}\right|,\left|\alpha-\frac{c_{e}-c_{i}}{2}-\frac{1}{2} \sqrt{\Delta(p)}\right|\right)>1 \\
\text { if } \Delta(p)<0: \alpha^{2}+\beta^{2}-\beta\left(c_{e}+c_{i}\right)+\alpha\left(c_{i}-c_{e}\right)>1
\end{array}\right.
$$

The proof of this property is given in Appendix B.

\section{Interpretation of these properties}

First, we may conclude from the demonstration of property 7 that when $(\mathrm{H})$ holds true, the network is equivalent to the following interaction diagram:

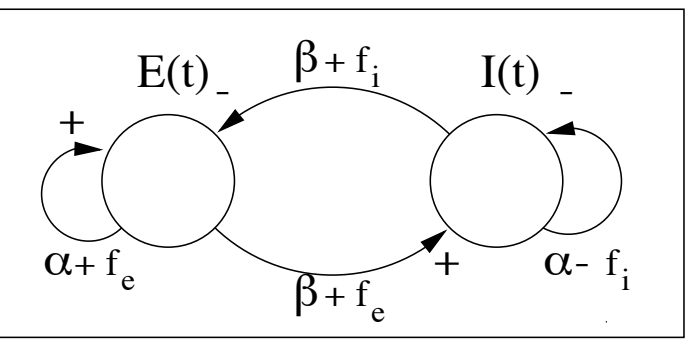

Figure 10 : Figure shows the equivalence between a microscopic description of a network of $N$ fully connected modules and a macroscopic description with a single module comprising one 
excitatory neuron $E(t)=\frac{1}{N} \sum_{i=1}^{N} x_{i}(t)$ and one inhibitory neuron $I(t)=\frac{1}{N} \sum_{i=1}^{N} y_{i}(t)$, where $x_{i}(t)$ and $y_{i}(t)$ are the respective activities of the excitatory neuron and of the inhibitory neuron of the ith module. In the microscopic description, the intramodule connections are equal to $\beta$ and $\alpha$, the connection between two neurons of different modules is $c_{e}$ when the connection is excitatory and $c_{i}$ when the connection is inhibitory. In the macroscopic description, $f_{i}=c_{i}(N-1)$ and $f_{e}=c_{e}(N-1)$. The signs indicate whether the connections in the direction of the arrows are excitatory or inhibitory.

If we wish to take a plausible approach, we have $\alpha-f_{i} \leq 0$, leading to a negative autocatalytic interaction for $\mathbf{I}(\mathbf{t})$. Furthermore, if we assume $f_{e} \approx f_{i}$, property 7 implies that the condition to be fulfilled is:

$$
\alpha^{2}+\beta^{2}+2 \beta f_{e}<1
$$

The size of the network is thus of major importance: drastic conditions must be imposed on the connections in order to obtain a naturally well desynchronized network. In addition, the criteria put forward in property 7 may be used to monitor the desynchronization speed. For instance, if $4 \beta\left(\beta+f_{e}+f_{i}\right)>\left(f_{e}-f_{i}\right)^{2}$, the desynchronization is monitored by $\alpha^{2}+\beta^{2}+f_{e}(\alpha+\beta)+f_{i}(\alpha-\beta)<$ 
1. The following graph is an illustration of this:
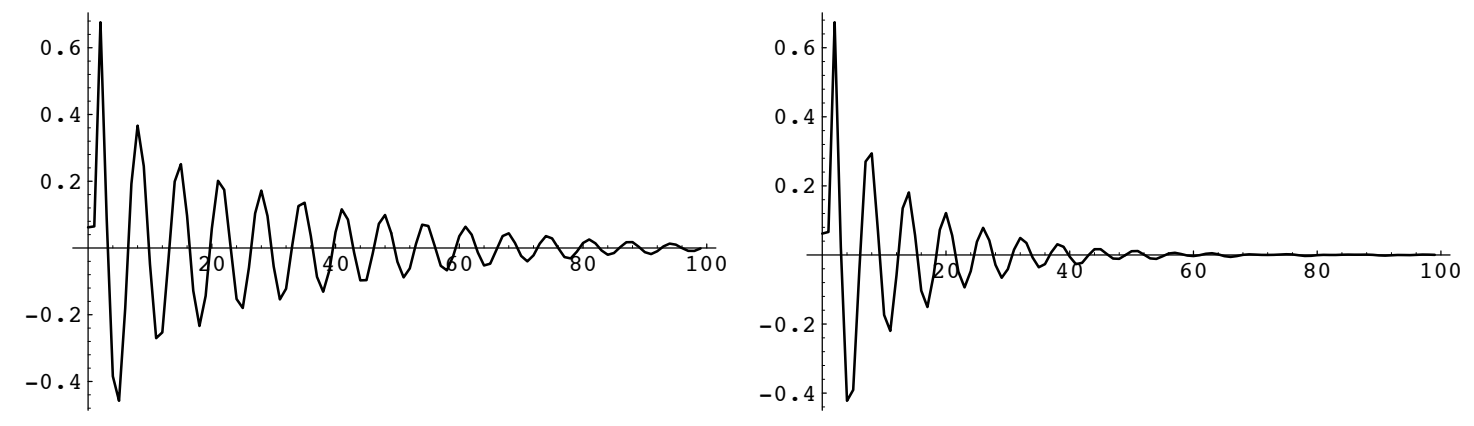

Figure 11: Figure $(A)$ represents $E(t)=\frac{1}{N} \sum_{i=1}^{N} x_{i}(t)$ with one hundred modules, $c_{e}=0.002$, $c_{i}=0.084, \alpha=0.9$ and $\beta=0.5$. Figure $(B)$ represents of $E(t)=\frac{1}{N} \sum_{i=1}^{N} x_{i}(t)$ with one hundred modules $, c_{e}=0.002, c_{i}=0.01, \alpha=0.9$ and $\beta=0.5$.

If we now look back to property 8 , we may see that that when one oscillator is a pacemaker, the others also have to be pacemakers. Numerically, this is still valid, even far from the secondary Hopf bifurcation. In addition, the necessary criterion to obtain at least one pacemaker neuron is independent of the size of the network.

Properties 8 and 9 are difficult to fulfill simultaneously. For instance, when $c_{e} \approx c_{i}$, the network must verify:

$$
\left\{\begin{aligned}
\alpha^{2}+\beta^{2}-2 c_{e} \beta & >1 \\
\alpha^{2}+\beta^{2}+2 \beta f_{e} & <1
\end{aligned}\right.
$$


These inequations obviously cannot be satisfied simultaneously. In order to enable the network to verify properties 8 and 9 , we must reduce the number of connections.

\subsubsection{Partially connected neural network}

Here, each oscillator, i, has been connected only to its neighbourhood V(i), comprising its eight nearest neighbours. We should first highlight the changes that are induced by these new connections, with respect to desynchronization. After this, we will investigate the possible changes that could be generated by a different discrete-time dynamic. With the connections diagram described above, and with the same kind of dynamic as that described in section 6.1, property 7 is changed into:

Property 9 Assume that the hypothesis (H) of section 6.2.2 holds true. The criterion stated in property 7 is still valid, for large networks, if we replace $f_{e}$ by $f_{\epsilon v}=8 c_{e}$ and $f_{i}$ by $f_{i v}=8 c_{i}$.

Proof: Because (H) holds true, we are near the secondary Hopf bifurcation. The evolution of $(\mathbf{E}(t), \mathbf{I}(t))$ is governed by:

$$
\begin{aligned}
& \mathbf{E}(t+1)=\alpha \mathbf{E}(t)-\beta \mathbf{I}(t)+\frac{1}{N}+\frac{c_{e}}{N} \underbrace{\sum_{i=1}^{N} \sum_{j \in V(i)} x_{j}(t)}_{S}-\frac{c_{i}}{N} \sum_{i=1}^{N} \sum_{j \in V(i)} y_{j}(t) \\
& \mathbf{I}(t+1)=\beta \mathbf{E}(t)+\alpha \mathbf{I}(t)+\frac{1}{N}+\frac{c_{e}}{N} \sum_{i=1}^{N} \sum_{j \in V(i)} x_{j}(t)-\frac{c_{i}}{N} \sum_{i=1}^{N} \sum_{j \in V(i)} y_{j}(t)
\end{aligned}
$$

A calculation distinguishing the different kinds of oscillators (oscillators of the center of the network, or those of the edge) yields:

$$
\frac{1}{N} S=8 \mathbf{E}(t)+O\left(\frac{1}{\sqrt{N}}\right)
$$


We can now deduce that the system satysfied by $(\mathbf{E}(t), \mathbf{I}(t))$ is as follows:

$$
\left\{\begin{array}{l}
\mathbf{E}(t+1)=\left(\alpha+8 c_{e}\right) \mathbf{E}(t)-\left(\beta+8 c_{i}\right) \mathbf{I}(t)+O\left(\frac{1}{\sqrt{N}}\right) \\
\mathbf{I}(t+1)=\left(\beta+8 c_{e}\right) \mathbf{E}(t)+\left(\alpha-8 c_{i}\right) \mathbf{I}(t)+O\left(\frac{1}{\sqrt{N}}\right)
\end{array}\right.
$$

For sufficiently large values of $\mathrm{N}$, the system above is similar to that we obtain in the proof of property 7 with $f_{e}$ replaced by $f_{e v}=8 c_{e}$ and $f_{i}$ by $f_{i v}=8 c_{i}$. The conclusions are similar to those for property 7

What is interesting in this property is that the desynchronization depends only on the neighbourhood, V(i), but not on the size, N, of the network, contrary to what is found with fully connected networks. Though it is easier to desynchronize a partially connected network than a fully connected one, maintaining a pacemaker activity while desynchronizing remains difficult. We will now make use of the observation made in section 3.1 in order to derive a new kind of evolution for network units. Indeed, oscillator evolution after synchronization gives:

$$
\left\{\begin{array}{l}
x_{i}(t+1)=\tanh _{\epsilon}\left(\alpha x_{i}(t)-\beta y_{i}(t)+\sum_{j \in V(i)}\left(c_{e} x_{i}(t)-c_{i} y_{j}(t)\right)\right) \\
y_{i}(t+1)=\tanh _{\epsilon}\left(\beta x_{i}(t)+\alpha y_{i}(t)+\sum_{j \in V(i)}\left(c_{e} x_{i}(t)-c_{i} y_{j}(t)\right)\right)
\end{array}\right.
$$

where $\tanh _{\epsilon}(u)$ is defined by: $\exists \eta / \tanh _{\epsilon}(u)=\frac{1-\epsilon^{2(1+\eta) u}}{1+\epsilon^{2(1+\eta))}}$.

We may assume that hypothesis $(\mathrm{H})$ of section 6.2 .2 still holds true. With this new kind of evolution, we are only able to produce simulations. A first observation is that it is really easy to obtain a pacemaker oscillator by using $\alpha^{2}+\beta^{2}>1$. After synchronization, desynchronization may be obtained for a range of parameters greater than that of property 9 . Figure 12 shows a configuration in which the network does not desynchronize, using the evolution model described 
in section 6.1 , but does with the above one.
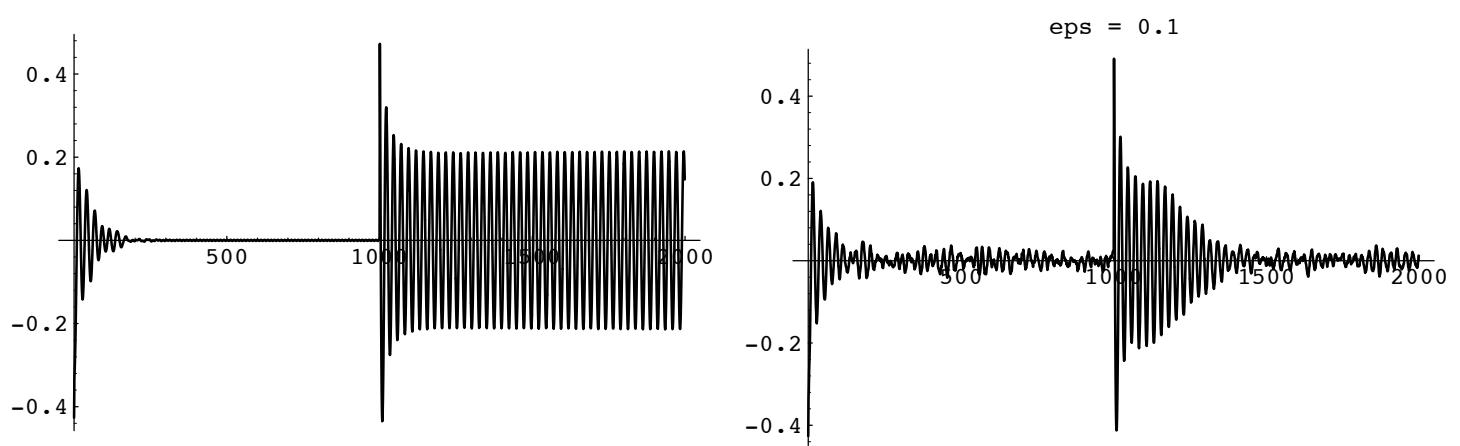

Figure 1D: In figure (A) we consider the representation of $E(t)=\frac{1}{N} \sum_{i=1}^{N} x_{i}(t)$ for values of paramaters which lead to good desynchronization of the system. We apply an input at $t=1000$ to each neuron of every module, the input being identical for each neuron. The modules remain synchronized even after some time. On the other hand, in figure(B) we consider a compact set valued iteration defined by a random value $\eta$ in $[-\epsilon, \epsilon], \epsilon=0.1$, and :

$$
\left\{\begin{array}{l}
x(t+1)=\tanh ((1+\eta)(\alpha x(t)-\beta y(t))) \\
y(t+1)=\tanh ((1+\eta)(\beta x(t)+\alpha x(t)))
\end{array}\right.
$$

Other parameters are identical to those of figure (A). The input is of the same kind as in figure (A). We observe a desynchronization. 


\section{Conclusion}

We have presented both a discrete-time version and a continuous-time version of the same neural oscillator. Throughout our study, we have attempted to show that the continuous version undergoes a bifurcation for some values of the parameters, while, the discrete-time model has two bifurcation points (it is well known that a discrete-time dynamic is more complex than its continuous counterpart). When parameters are chosen appropriately, both models exhibit oscillatory behaviors. With such values of the parameters, we have put forward a relationship between the models at the birth of oscillations. We have then investigated monitoring of the phase lag between oscillators, through mutual connections. For the continuous time model, we obtained some analytical results, which are in accordance with those obtained by Hoppensteadt and Izhikevitch (1997), when the coupling consists of one connection. These results are extended to several connections by the use of numerical simulations.

In contrast, the discrete-time model does not allow such an analysis. In any case, it was observed that it becomes difficult to assess the phase lag for a network of more than two oscillators, with respect to the continuous model. For this reason, we have decided to achieve synchronization in larger networks by means of inputs.

Then, we have investigated the desynchronization of synchronized networks, but only for a discrete-time dynamic. This has seldom been done before. We have therefore carried out a study near the secondary Hopf bifurcation point for the discrete-time model. We have managed to obtain analytical results which emphasize that fully connected networks are not relevant to any 
modelling of desynchronization, since they impose overly drastic conditions on the connections. Conversely, a sparser connected network allows us to maintain an oscillatory activity after stimulation, as well as a weak amplitude for the mean activity. Secondly, we also notice that macroscopic description is possible for such a network. This may be an advantage from a biological point of view, in that recordings of electrical activity cannot be achieved for a single neuron. Only the activity of a network is accessible.

From this point of view it may be seen that this model, which allows us to switch from a microscopic to a macroscopic description, may prove to be of value. Finally, we made an attempt, with a new kind of evolution model, which has given promising simulation results, with respect to desynchronization, but so far lacks any analytical results. Further investigations should be carried out in this direction, in order to work towards biological plausibility. 


\section{APPENDIX A}

Property 7 Let us call $f_{e}=c_{e}(N-1)$ and $f_{i}=c_{i}(N-1)$ these are the excitatory and the inhibitory strengths of the network, respectively.

Using $\Delta(q)=\left(f_{e}-f_{i}\right)^{2}-4 \beta\left(\beta+f_{e}+f_{i}\right)$, we need :

$$
\left\{\begin{array}{l}
\text { if } \Delta(q)>0: \max \left(\left|\alpha+\frac{f_{e}-f_{i}}{2}+\frac{1}{2} \sqrt{\Delta(q)}\right|,\left|\alpha+\frac{f_{e}-f_{i}}{2}-\frac{1}{2} \sqrt{\Delta(q)}\right|\right)>1 \\
\text { if } \Delta(q)<0: \alpha^{2}+\beta^{2}+f_{e}(\alpha+\beta)+f_{i}(\beta-\alpha)<1
\end{array}\right.
$$

Proof : Near the secondary Hopf bifurcation, the dynamic is governed by :

$$
\left\{\begin{array}{l}
x_{i}(t+1)=\alpha_{i} x_{i}(t)-\beta_{i} y_{i}(t)+\sum_{j=1, j \neq i}^{N}\left(c_{e} x_{j}(t)-c_{i} y_{j}(t)\right) \\
y_{i}(t+1)=\beta_{i} x_{i}(t)+\alpha_{i} y_{i}(t)+\sum_{j=1, j \neq i}^{N}\left(c_{e} x_{j}(t)-c_{i} y_{j}(t)\right)
\end{array}\right.
$$

Since the oscillators are assumed to be identical, we write $\alpha_{i}=\alpha$ and $\beta_{i}=\beta$ which implies that the activities of the excitatory and of the inhibitory neurons evolve as follows:

$$
\left\{\begin{array}{l}
\mathbf{E}(t+1)=\left(\alpha+c_{e}(N-1)\right) \mathbf{E}(t)-\left(\beta+c_{i}(N-1)\right) \mathbf{I}(t) \\
\mathbf{I}(t+1)=\left(\beta+c_{e}(N-1)\right) \mathbf{E}(t)+\left(\alpha-c_{i}(N-1)\right) \mathbf{I}(t)
\end{array}\right.
$$

Using $f_{e}=c_{e}(N-1)$ and $f_{i}=c_{i}(N-1)$ we rewrite the system in the form :

$$
\left\{\begin{array}{l}
\mathbf{E}(t+1)=\left(\alpha+f_{e}\right) \mathbf{E}(t)-\left(\beta+f_{i}\right) \mathbf{I}(t) \\
\mathbf{I}(t+1)=\left(\beta+f_{e}\right) \mathbf{E}(t)+\left(\alpha-f_{i}\right) \mathbf{I}(t)
\end{array}\right.
$$

The characteristic polynom of the evolution matrix is :

$$
q(x)=x^{2}-\left(2 \alpha-f_{i}+f_{e}\right) x+\alpha^{2}+\beta^{2}+\alpha\left(f_{e}-f_{i}\right)+\beta\left(f_{i}+f_{e}\right)
$$


Using its discriminant :

$$
\Delta(q)=\left(f_{e}-f_{i}\right)^{2}-4 \beta\left(\beta+f_{e}+f_{i}\right)
$$

we are led to the following conclusions:

1. if $\Delta(q)<0$

We have two conjuguate eigenvalues, $v p 1$ and $v p 2$. The condition required for $(0,0)$ to be an attractor is thus:

$$
|v p 1|=|v p 2|=\alpha^{2}+\beta^{2}+f_{e}(\alpha+\beta)+f_{i}(\beta-\alpha)<1
$$

2. if $\Delta(q)>0$

the condition required for $(0,0)$ to be an attractor is

$$
\max \left(\left|v p_{1}\right|,\left|v p_{2}\right|\right)<1
$$

where :

$$
\begin{gathered}
v p_{1}=\alpha+\frac{f_{e}-f_{i}}{2}+\sqrt{\left(\frac{f_{e}-f_{i}}{2}\right)^{2}-\beta\left(\beta+f_{e}+f_{i}\right)} \\
v p_{2}=\alpha+\frac{f_{e}-f_{i}}{2}-\sqrt{\left(\frac{f_{e}-f_{i}}{2}\right)^{2}-\beta\left(\beta+f_{e}+f_{i}\right)}
\end{gathered}
$$




\section{APPENDIX B}

Property 8 If the network satisfies property 7 , using $\Delta(p)=4 \beta\left(c_{i}+c_{e}-\beta\right)+\left(c_{e}-c_{i}\right)^{2}$ we need :

$$
\begin{cases}\text { if } \Delta(p)>0: & \max \left(\left|\alpha-\frac{c_{e}-c_{i}}{2}+\frac{1}{2} \sqrt{\Delta(p)}\right|,\left|\alpha-\frac{c_{e}-c_{i}}{2}-\frac{1}{2} \sqrt{\Delta(p)}\right|\right)>1 \\ \text { if } \Delta(p)<0: & \alpha^{2}+\beta^{2}-\beta\left(c_{e}+c_{i}\right)+\alpha\left(c_{i}-c_{e}\right)>1\end{cases}
$$

Proof : For the sake of simplicity, we denote $\left(x_{1}(t), y_{1}(t), \ldots, x_{N}(t), y_{N}(t)\right)$ by $z(t)$ where $N$ is the number of oscillator. Since $(H)$ holds true, the dynamic of $z(t)$ is governed by :

$$
z(t+1)=D F(0) z(t)
$$

where :

$$
D F(0)=\left(\begin{array}{cccccc}
\alpha & -\beta & c_{e} & -c_{i} & c_{e} & -c_{i} \\
\beta & \alpha & c_{e} & \vdots & \vdots & \vdots \\
c_{e} & -c_{i} & \ddots & -c_{i} & \vdots & \vdots \\
\vdots & \vdots & \ldots & \ddots & c_{e} & -c_{i} \\
\vdots & \vdots & \ldots & \ldots & \alpha & -\beta \\
c_{e} & -c_{i} & \ldots & \ldots & \beta & \alpha
\end{array}\right)
$$

The condition we are looking for is $\rho(D F(0))>1$, where $\rho(M)=\max _{\alpha \in S_{p}(M)}|\alpha|$. If we call $\mathrm{N}$ the number of oscillators, let $P_{N}$ be the following polynom :

$$
P_{N}(x)=\operatorname{det}\left(D F(0)-x I_{2 N}\right)
$$

Our aim is to determine the roots of this polynom which are the eigenvalues of $D F(0)$. Simple calculations lead to the recurrence formula : 
if $N \geqslant 2$ :

$$
P_{N}(x)=p(x)\left[P_{N-1}(x)+p^{N-2}(x)\left(\left(c_{i}-c_{e}\right) x+\left(c_{e}-c_{i}\right) \alpha+\left(c_{e}+c_{i}\right) \beta\right)\right]
$$

where

$$
p(x)=x^{2}+\left(c_{e}-c_{i}-2 \alpha\right) x+\alpha^{2}+\beta^{2}-\alpha\left(c_{e}-c_{i}\right)+\beta\left(-c_{i}-c_{e}\right)
$$

and if $N=1$ :

$$
P_{1}(x)=x^{2}-2 \alpha x+\alpha^{2}+\beta^{2}
$$

This finally implies that:

$$
P_{N}(x)=p^{N-1}(x) q(x)
$$

where $q(x)$ is the polynom introduced in the demonstration of property 7 .

$$
\begin{aligned}
& \Delta(p)=\quad 4 \beta\left(c_{i}+c_{e}-\beta\right)+\left(c_{e}-c_{i}\right)^{2} \\
& \Delta(q)=-4 \beta\left(\beta+(N-1)\left(c_{e}+c_{i}\right)\right)+(N-1)^{2}\left(c_{e}-c_{i}\right)^{2}
\end{aligned}
$$

\section{A ) conditions for $p$}

The following study leads to conditions that are independent of the size of the system

1. $4 \beta\left(c_{i}+c_{e}-\beta\right)+\left(c_{e}-c_{i}\right)^{2}<0$

In this case, we have two conjugate eigenvalues of order $\mathrm{N}-1$. To get at least one eigenvalue with its module greater than one we need to have :

$$
\alpha^{2}+\beta^{2}-\beta\left(c_{e}+c_{i}\right)+\alpha\left(c_{i}-c_{e}\right)>1
$$

When $c_{i}=c_{e}$ we must have $\alpha^{2}+\beta^{2}-2 c_{e} \beta>1$ 
2. $4 \beta\left(c_{i}+c_{e}-\beta\right)+\left(c_{e}-c_{i}\right)^{2}>0$

We have two real eigenvalues, $v_{1}, v_{2}$ which are :

$$
\alpha-\frac{c_{e}-c_{i}}{2} \pm \sqrt{\beta\left(c_{i}+c_{e}-\beta\right)+\left(\frac{c_{e}-c_{i}}{2}\right)^{2}}
$$

The condition we were looking for is as follows :

$$
\max \left(\left|v_{1}\right|,\left|v_{2}\right|\right)>1
$$

If $c_{i}=c_{e}$ the eigenvalues are equal to $\alpha \pm \sqrt{\beta\left(2 c_{e}-\beta\right)}$

and the condition becomes:

$$
\alpha>1-\sqrt{\left(\beta\left(2 c_{e}-\beta\right)\right)}
$$

B ) conditions for $\mathbf{q}$ The same study can be carried changing $c_{e}$ into $f_{e}=c_{e}(N-1)$ and $c_{i}$ into $f_{i}=c_{i}(N-1)$. The calculation leads to the conditions required for the activity of the excitatory and the inhibitory neurons not to tending towards $(0,0)$. Therefore, the roots of $q$ cannot be bigger than 1 , since we have assumed that the network verifies property 7 .I 


\section{References}

Atiya, A., \& Baldi, P. (1989). Oscillations and synchronizations in neural networks : an exploration of the labelling hypothesis. International Journal of Neural Systems, 1, 103-124.

Borisyuk, R. M., \& Kirilov, A. B. (1992). Bifurcation analysis of a neural network model. Biological Cybernetics, 66, 319-325.

Chapeau-Blondeau, F., \& Chauvet, G. (1992). Stable, oscillatory and chaotic regimes in the dynamics of small neural oscillators with delay. Neural Network, 5, 735-743.

Demongeot, J., Kulesa, P., \& Murray, J. D. (1997). Compact set valued flows II : applications in biological modelling. C. R. Acad. Sci., 324, series II b, 107-115.

Ermentrout, B., \& Kopell, N. (1994). Learning of phase lags in coupled oscillators. Neural Computation, 6, 225-241.

Gluck, M. A. (1996). Computational models of hippocampal function in memory. Hippocampus, 6. New-York : Wiley-Liss.

Gray, C. M. \& Singer, W. (1989). Stimulus-specific neural oscillations in orientation of cat visual cortex. Proceedings of the national Academy of Sciences, USA, 86, 1698-1702.

Guckenheimer, J. (1975). Isochrons and phaseless sets. Journal of Mathematical Biology, 2, $259-273$.

Hoppensteadt, F. C. and Izhikevich, E. M. (1997). Weakly connected neural networks. In J. E. Marsden, L. Sirovitch \& F.John,Synaptic Organizations of the Brain(pp. 353-379). New-York: Springer-Verlag. 
Li, Z., \& Hopfield, J. J. (1989). Modeling the olfactory bulb and its neural oscillatory processing. Biological Cybernetics, 61, 379-392.

Pasemann, F. (1995). Characterization of periodic attractors in neural ring network. Neural Network, 8, 421-429.

Pham Dinh, T., \& Demongeot, J. (1983). Simulation of a biological oscillator : the respiratory system. Journal of Theorical Biology, 103, 113-132

Thomas, R., \& D'Ari, R. (1990). Biological Feedback. Boston : CRC Press.

Von der Malsburg, C. (1981).Models of Neural Networks II.In E. Domany, J. L. van Hemmen \& K. Schulten, chapter 2. Berlin: Springer-Verlag.

Wang, D. L. (1996). Synchronous oscillations based on lateral connections. In J.Sirosh , R.Miikkulainen \& Y. Choe (Eds.). Lateral connections in the cortex : structure and function.

Winfree, A. T. (1974). Patterns of phase compromise in biological cycles.Journal of Mathematical Biology, 1, 73-95.

Wilson, H. R., \& Cowan, J. D. (1972). Excitatory and inhibitory interactions in localized populations of model neurons. Biophysical Journal, 12, 1-24. 